\title{
A Long-Term Control of Intramedullary Thoracic Spinal Cord Metastasis from Small Cell Lung Cancer
}

\author{
Hajime Osawa, Shinichiro Okauchi, Gen Ohara, Katsunori Kagohashi, Hiroaki Satoh*
}

\begin{abstract}
Radiotherapy with systemic corticosteroid therapy has been used to treat intramedullary spinal cord metastasis (ISCM), but recovery of function and long-term survival of these patients has been rarely observed. We report herein a small cell lung cancer (SCLC) patient with recurrent thoracic ISCM, who was successfully treated with radiotherapy and systemic corticosteroid therapy. A 70-year-old man, who was diagnosed as having SCLC seven months previously, developed thoracic ISCM. Soon after the detection of the lesion, the patient received radiotherapy with systemic corticosteroid therapy. Sensory disturbance in both extremities and neurogenic bladder and bowel dysfunction was recovered. The patient could walk after irradiation again. The patient received additional chemotherapy and survived 20 months after the diagnosis of ISCM recurrence. Prompt diagnosis and appropriate treatment for ISCM and effective chemotherapy for recurrent SCLC might be the favorable factors for such patients. Further studies will be required to define a favorable subset of patients most likely to benefit from a conventional approach.
\end{abstract}

\section{KEYWORDS}

long-term control; intramedullary spinal cord metastasis; small cell lung cancer

\section{AUTHOR AFFILIATIONS}

Division of Respiratory Medicine, Mito Medical Center, University of Tsukuba

* Corresponding author: Division of Respiratory Medicine, Mito Medical Center, University of Tsukuba, Miya-machi 3-2-7, Mito, 305-8575, Ibaraki, Japan; e-mail: hirosato@md.tsukuba.ac.jp

Published online: 14 September 2018

Acta Medica (Hradec Králové) 2018; 61(2): 57-59

https://doi.org/10.14712/18059694.2018.52

(c) 2018 The Authors. This is an open-access article distributed under the terms of the Creative Commons Attribution License (http://creativecommons.org/licenses/by/4.0), which permits unrestricted use, distribution, and reproduction in any medium, provided the original author and source are credited. 


\section{INTRODUCTION}

Intramedullary spinal cord metastasis (ISCM) is a rare but dismal metastasis, and it has been reported in $0.9-2.1 \%$ of all neoplasm autopsies (1-4). Lung and breast carcinomas comprise the most common primary tumor sites in ISCM. Among them, small cell lung cancer (SCLC) is the most common histological type that develop $\operatorname{ISCM}(3,5,6)$. ISCM is not only associated with severe pain, but also with paralysis, sensory loss, urinary and fecal incontinency, i.e. neurogenic bladder and bowel dysfunction (7-9), when the neurologic elements are compressed. Many patients with intramedullary metastasis whose treatment is not successful suffer from these symptoms that obviously degrade the quality of life. If appropriate treatment cannot be made in time, neuropathy is irreversible even if metastatic lesions shrink. At present, ISCM lacks well-defined treatment guidelines, but rapid diagnosis and appropriate treatment can improve both the quality and length of remaining life. Surgically resectable patients are rare, and irradiation of the metastatic lesion and corticosteroid therapy are usually carried out. Unfortunately, however, complete relief of the symptoms and improvements upon radiological evaluations are rarely expected. Overall prognosis of patients with ISCM is poor (1-3), although a multimodality approach may contribute to the improvement of quality of life and survival.

We show herein a SCLC patient with thoracic ISCM who was successfully treated with a combination of radiotherapy, corticosteroids, and chemotherapy. He experienced relief of the symptoms and showed improvements upon subsequent radiological evaluations. The patient survived 20 months after the diagnosis of ISCM recurrence.

\section{CASE REPORT}

A 70-year-old man was admitted to our hospital with weakness and dysesthesia of the bilateral lower extremities, and urinary and fecal incontinence. He was a current smoker. Seven months previously, he was diagnosed as having SCLC in the lower lobe of the left lung (Figure 1). As there was no distant metastasis, he received platinum-containing chemoradiotherapy. Two months after the therapy, he noticed weakness and dysesthesia in left lower extremity, which was rapidly progressing to both lower extremities. Urinary and fecal incontinence was gradually progressed over a month. On admission to our hospital, he was unable to walk. Magnetic resonance imaging (MRI) revealed thoracic ISCM at Th11 (Figure 2-A). Weakness and dysesthesia in both lower extremities, and urinary and fecal incontinence, which was diagnosed as neurogenic bladder and bowel dysfunction, were symptoms due to intramedullary metastasis. As the primary lesion was controlled and no other metastatic site was found, therapy for the ISCM could be started soon after the detection of the ISCM. The patient was treated with dexamethasone at an initial dose of $8 \mathrm{mg}$ per day for 14 days followed by gradual tapering off over the next 2 weeks.

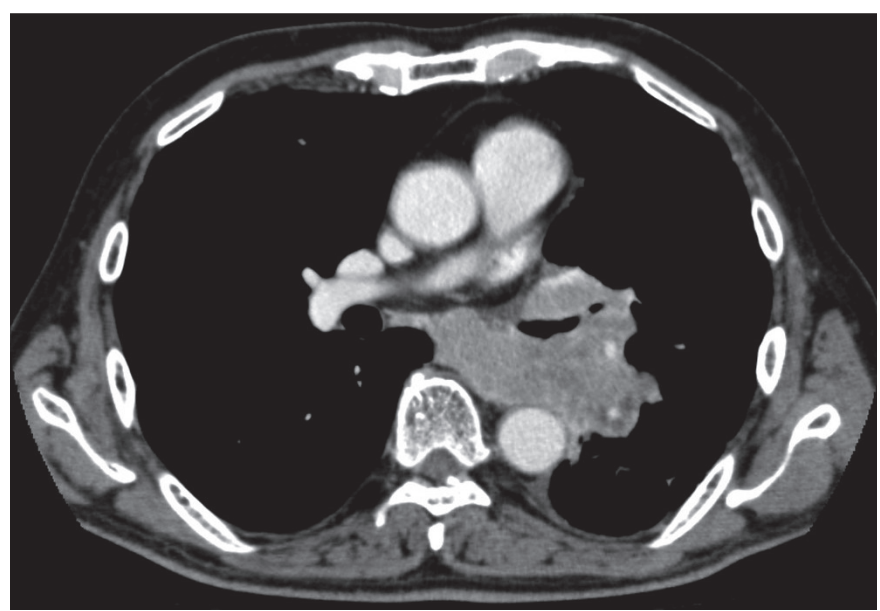

Fig. 1 Chest CT scan at the time of diagnosis of small cell lung cancer.

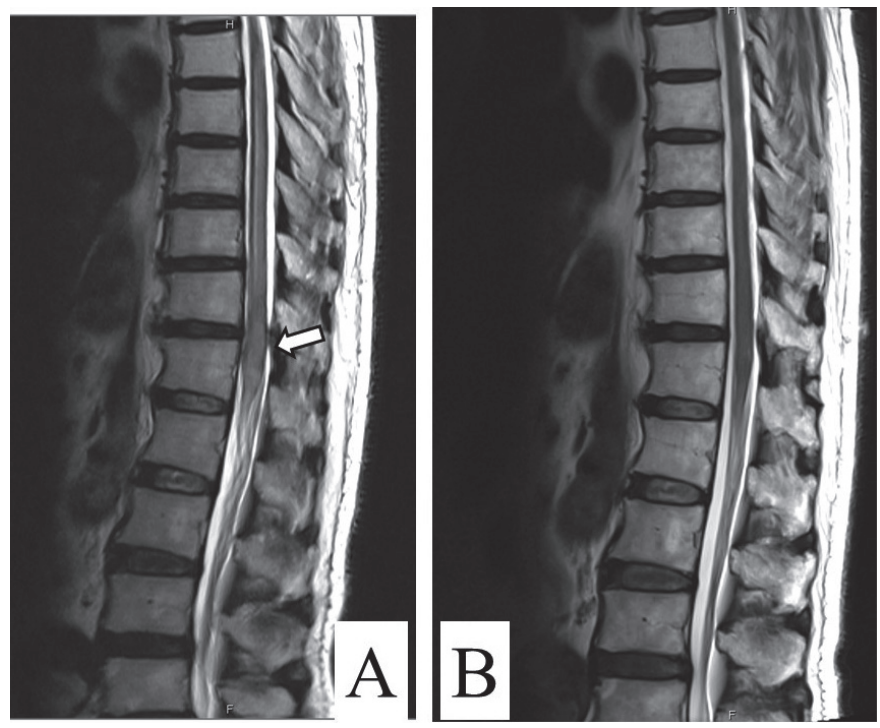

Fig. 2 Magnetic resonance imaging (MRI) of the thoracic spinal intramedullary metastasis. An intramedullary spinal cord tumor with adjacent edema can be observed at the Th11 level on a T2weighted image (arrow) (A). The follow-up MRI 2 months after the initiation of irradiation showed a disappearance of the intramedullary spinal cord tumor and adjacent edema at the Th11 level on a T2-weighted image (B).

He received palliative irradiation for the ISCM lesion up to $40 \mathrm{~Gy}$ (16 fractions). At the follow-up 3 weeks after the initiation of dexamethasone and radiotherapy, the neurologic status showed distinct improvement insofar as the patient was able to walk a short distance on his own. Improvement of urinary and fecal incontinence was also observed. The follow-up MRI 2 months after the initiation of irradiation showed a significant decrease in the size of the ISCM (Figure 2-B). Thereafter, the patient received 6 courses of platinum-containing chemotherapy, 5 courses of amrubicin, and 2 courses of irinotecan therapy. He was regularly monitored in our outpatient division. He could walk for 18 months but died of SCLC 20 months after the diagnosis of ISCM recurrence. 


\section{DISCUSSION}

With early diagnosis and appropriate treatment, selected patients with ISCM may benefit from improved neurological outcome and long-term survival, as observed in the present case. In most patients with ISCM, however, treatment is not successful, and they suffer from the symptoms that obviously degrade the quality of life. Even effective, treatment delay can lead to irreversible neuropathy. An unfavorably clinical course in most patients leads to debilitating and disastrous outcomes. Therefore, ISCM is a medical emergency with potentially debilitating and disastrous outcomes (10).

Immediate treatment with irradiation of the ISCM and corticosteroid therapy is the most commonly used option $(1-4,11)$. Hashii et al reported that $56 \%$ of the patients who received radiotherapy showed either neurological improvement or pain alleviation (12). However, other previous reports described that many patients receive these therapies, but rarely with remarkable effect $(1-4,11)$. In a review by Winkelman (10), clinical evidence for the effectiveness of irradiation of the ISCM and corticosteroid therapy was presented, however, neither shrinkage of the metastatic lesion on MRI nor improvement of impaired symptoms were rarely observed.

Surgery remains the treatment of choice for symptomatic intramedullary lesions, but the optimal management of intramedullary metastasis is still controversial. Despite of recent advances in surgical management of intramedullary metastasis, high complication rate causes clinicians to hesitate to perform surgical resection. Corticosteroids have the effect of reducing edema around the metastatic site. However, no standardized method has been established for the type of administered drug, dosage and administration period. Radiotherapy has an important role, particularly in treatment of radiosensitive tumors and in patients who are not candidates for surgery. Novel approaches such as stereotactic radiosurgery can be promising (13-15). With regard to radiotherapy, standard irradiation method was not established. Recently, stereotactic radiotherapy has attached attention $(14,15)$. This radiotherapy can be a safe and effective option in selected cases, and is attractive that this radiotherapy may preserve neurological function $(14,15)$. Radiosensitive tumors may be the most suitable for this radiotherapy.

It is unknown why this patient achieved a good therapeutic effect. The following factors are considered as the reasons of good therapeutic effect. 1) Correct diagnosis was possible within a week from the admission of the patient, though it is difficult to judge onset of ISCM. 2) Multidisciplinary treatments could be started in a week after the detection of the ISCM. 3) At the same time as irradiation, dexamethasone was continued without reducing the dose for 2 weeks. 4) The presence of effective antitumor drugs had important role for long-term control of ISCM.

Since ISCM is debilitating and disastrous metastasis, chest physicians need to be aware of this metastasis. MRI is essential for accurate diagnosis. If there are symptoms suspected of this metastasis, MRI should be performed immediately. Shortening the interval from the appearance of symptoms to the start of treatment is related to the therapeutic effect, though "the prime-time" is beyond our knowledge. To achieve long-term survival, in addition, it is essential to control not only ISCM but also the other lesions than the ISCM. Presence of effective chemotherapeutic drugs is another key factor. Rehabilitation that improves symptoms may also be important. With early diagnosis and appropriate treatment, selected patients can achieve improved neurological outcome and longterm survival. The therapeutic environment in which these multidisciplinary treatments are available and the physical condition of the patient that can withstand these treatments can be circumstances indispensable to achieve successful outcome. It is important to accumulate knowledge and to develop appropriate therapies.

\section{CONFLICT OF INTEREST}

None.

\section{FUNDING SOURCE}

None.

\section{REFERENCES}

1. Costigan DA, Winkelman MD. Intramedullary spinal cord metastasis. A clinicopathological study of 13 cases. J Neurosurg 1985; 62(2): 227-33.

2. Lee SS, Kim MK, Sym SJ, et al. Intramedullary spinal cord metastases: a single-institution experience. J Neurooncol 2007; 84(1): 85-9.

3. Kalayci M, Cagavi F, Gul S, Yenidunya S, Acikgoz B. Intramedullary spinal cord metastases: diagnosis and treatment: an illustrated review. Acta Neurochir (Wien) 2004; 146(12): 1347-54.

4. Okamoto H, Shinkai T, Matsuno Y, et al. Intradural parenchymal involvement in the spinal subarachnoid space associated with primary lung cancer. Cancer 1993; 72(9): 2583-88.

5. Katsenos S, Nikolopoulou M. Intramedullary thoracic spinal metastasis from small-cell lung cancer. Monaldi Arch Chest Dis 2013; 79(3-4): 140-2.

6. Koutsis G, Spengos K, Potagas C, Dimitrakopoulos A, Sfagos K, Zakopoulos N. Intramedullary spinal cord metastases in a patient with small-cell lung cancer. Eur J Intern Med 2006; 17(5): 372-4.

7. Parker AL, Pugh T, Hirsch MA. A rare intramedullary spinal cord metastasis from a retroperitoneal leiomyosarcoma presenting as a non-traumatic spinal cord injury. Am J Phys Med Rehabil 2017; 96(7): e134-7.

8. Fujimoto N, Hiraki A, Ueoka H, et al. Intramedullary spinal cord recurrence after high-dose chemotherapy and autologous peripheral blood progenitor cell transplantation for limited-disease small cell lung cancer. Lung Cancer 2000; 30(2): 145-8.

9. Wilson PE, Oleszek JL, Clayton GH. Pediatric spinal cord tumors and masses. J Spinal Cord Med 2007; 30(Suppl 1): S15-20.

10. Winkelman MD, Adelstein DJ, Karlins NL. Intramedullary spinal cord metastasis. Diagnostic and therapeutic considerations. Arch Neurol 1987; 44(5): 526-31.

11. Hickey M, Farrokhyar F, Deheshi B, Turcotte R, Ghert M. A systematic review and meta-analysis of intralesional versus wide resection for intramedullary grade I chondrosarcoma of the extremities. Ann Surg Oncol 2011; 18(6): 1705-9.

12. Hashii H, Mizumoto M, Kanemoto A, et al. Radiotherapy for patients with symptomatic intramedullary spinal cord metastasis. J Radiat Res 2011; 52(5): 641-55.

13. Mut M, Schiff D, Shaffrey ME. Metastasis to nervous system: spinal epidural and intramedullary metastases. J Neurooncol 2005; 75(1): 43-56.

14. Kaal EC, Vecht CJ. CNS complications of breast cancer: current and emerging treatment options. CNS Drugs 2007; 21(7): 559-79.

15. Hernández-Durán S, Hanft S, Komotar RJ, Manzano GR. The role of stereotactic radiosurgery in the treatment of intramedullary spinal cord neoplasms: a systematic literature review. Neurosurg Rev 2016; 39(2): 175-83. 